\title{
Perfil epidemiológico da demanda em unidades de emergência hospitalar: uma revisão de literatura
}

\author{
Epidemiological profile of demand on hospital emergency units: a literature review \\ Perfil epidemiológico de la demanda en unidades hospitalarias de emergencia: una \\ revisión de literatura
}

Juliana Andrade Pereira ${ }^{1 *}$, João Pedro Paulino Ruas², Victor de Oliveira Flausino ${ }^{2}$, Ana Flavia Nunes Barbosa ${ }^{2}$, Thiago Vinícius dos Santos Ferreira², Tércio Cirqueira Correia ${ }^{2}$, Júlia Mendes

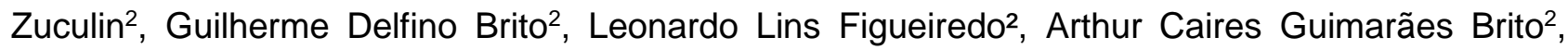
Bruna Afonso Lopes Lima², Maria Thereza Meira Araujo², Anna Cecília Castro e Abreu².

\section{RESUMO}

Objetivo: Descrever o perfil da demanda em Unidades de Emergência Hospitalar pela revisão de literatura. Métodos A busca de dados abrangeu nas bases de dados Biblioteca Virtual de Saúde (BVS), Literatura Latino-Americana e do Caribe em Ciências da Saúde (LILACS) e Scientific Electronic Library Online (SciELO). Os critérios de inclusão dos artigos foram: estudos na íntegra, no idioma português, no ano de 2000 a 2019 e que contemplassem o tema da pesquisa. Resultados: Verificou-se que a maioria dos pacientes nas Unidades de Emergência Hospitalar era do sexo masculino e da faixa etária de 20 a 39 anos e de 40 a 59 anos. Quanto ao perfil clínico identificou-se que as principais queixas eram por febre, tosse, vômitos, alergias, dores abdominais, diarreias, dor de ouvidor e de garganta, cefaleia, acidentes de trânsitos e problemas cardiovasculares. Conclusão: Salienta-se que estes atendimentos mostram que as sinais e sintomas são de baixa complexidade pela avaliação de Risco de Manchester, mostrando que a maioria dos atendimentos poderia ser solucionada nas atenção primária, o que contribuiria para diminuição das filas nas salas de espera e maior organização da rede de atenção à saúde relacionada às urgências.

Palavras-chave: Emergência, Urgência, Avaliação.

\section{ABSTRACT}

Objective: To describe the profile of demand on hospital emergency units by the literature review. Methods: The data included in the databases of Virtual Health Library (BVS), Latin American literature and Caribbean Health Sciences (LILACS) and Scientific Electronic Library Online (SciELO). The criteria for inclusion of articles were: studies in their entirety, in the Portuguese language, in the year 2000 to 2019 and that favored the research theme. Results: It was found that most of the patients in hospital emergency units was male and the age group of 20 to 39 years and 40 to 59 years. As the clinical profile, identified that the main complaints were for fever, cough, vomiting, abdominal pains, allergies, diarrhea, sore throat, headache and Ombudsman, transit accidents and cardiovascular problems. Conclusion: It should be noted that these calls show that the signs and symptoms are of low complexity for the risk assessment of Manchester, showing that the majority of attendances could be resolved in primary care, which would contribute to decrease of the queues in waiting rooms and largest organization of network of health care related to the emergency room.

Keywords: Emergency, Urgency, Evaluation.

1Faculdades Unidas do Norte de Minas, Universidade Estadual de Montes Claros - Unimontes, Universidade Federal dos Vales do Jequitinhonha e Mucuri- UFVJM Montes Claros-Minas Gerais. *E-mail: juhorientadora@gmail.com

2 UNIFIPMoc - Centro Universitário FIPMoc. Montes Claros-Minas Gerais. 


\section{RESUMEN}

Objetivo: Describir el perfil de la demanda en hospital revisión de unidades de emergencia de la literatura. Métodos: De losdatosincluidos en las bases de datos de Biblioteca Virtual en salud (BVS), literatura latinoamericana y Caribe Health Sciences (LILACS) y Scientific Electronic Library Online (SciELO). Los criterios de inclusión de los artículos fueron: estudios en sutotalidad, en lalengua portuguesa, en el año 2000 a 2019 y esofavorecieron el tema de investigación. Resultados: Se encontró que lamayoría de los pacientes en unidades de emergencia de hospital era masculino y laedad del grupo de 20 a 39 años y 40 a 59 años. Como el perfil clínico, identificaron que lasprincipalesquejasfueronfiebre, tos, vómitos, abdominal dolores, alergias, diarrea, dolor de garganta, dolor de cabeza y defensor del pueblo, problemas cardiovasculares y accidentes de tránsito.Conclusión: Cabe destacar que estas llamadasmuestran que los signos y síntomas de baja complejidad para la evaluación del riesgo de Manchester, que muestra que lamayoría de asistenciaspodríaresolverse en atención primaria, que contribuiría a disminución de las colas en salas de espera y laorganización más grande de lared de atención de lasalud relacionadas conla sala de emergencias.

Palabras clave: Emergencia, Urgente, Evaluación.

\section{INTRODUÇÃO}

Observando que o hospital dispõe de um serviço de emergência que funciona durante 24 horas ininterruptas, com umas equipes multiprofissionais qualificada em várias áreas de emergências clínicas e traumáticas, acredita-se que os indivíduos recorram a este serviço por razões diversas: funcionamento durante 24 horas, fácil acessibilidade, impossibilidade de recorrer aos serviços ambulatoriais (SILVA GL e RABINOVICH EP, 2013). O Pronto socorro é um local destinado para presta assistência ao paciente que se encontra em estado de urgência ou emergência.

O conhecimento do perfil da clientela atendida é de suma importância para o melhoramento do serviço prestado facilitando o direcionamento de políticas pública. O perfil geral das vítimas de trauma deste estudo foram predominantemente pacientes do sexo masculino, adultos jovens, com maior número de ocorrências nos meses de Fevereiro, Abril e Maio, meses nos quais há datas festivas, atendidas em vias públicas urbanas e rodovias. Os procedimentos realizados estão de acordo com os protocolos nacionais e internacionais de trauma e foram específicos para as necessidades de cada indivíduo. Tais dados apresentam como reflexo, o alto custo para a sociedade e para o SUS (PRAÇA WR et al., 2017).

Devido ao elevado crescimento do número de atendimentos de urgência e emergência (AUE) o Brasil, gerados pela violência, acidentes de Trânsito e doenças de várias etiologias, sobretudo cardiovasculares, torna-se necessário um atendimento rápido e especializado a fim de prestar os primeiros socorros aos usuários vítimas de traumas e males súbitos, ainda na cena do fato. Essa assistência conta, ainda, com equipe qualificada para o atendimento, o que contribui na redução do número de óbitos e suas complicações (MALVESTIO MAA e SOUSA RMC, 2002).

Essas vítimas são encaminhadas aos diversos centros de emergência, locais apropriados para o atendimento desses usuários, contando com equipe especializada e equipamentos adequados para tal finalidade (ZIMMERMANN LP et al.; 2011).

Uma denominação desse tipo de serviço é o Pronto Socorro (PS), que além de executar atendimento aos clientes em situações de agravo à saúde, com ameaça iminente de morte, de alta complexidade e variedade, tornou-se um local em que os clientes ficam internados por muitos dias (GARLET ER et al.; 2009).

Segundo Olivati FN, et al. (2010), as principais causas de procuras do PS foram casos de risco para os clientes, em que as relevantes queixas foram agudas e envolveram incômodo físico, compondo um sinal característico de parâmetros terapêuticos de atenção primaria além de problemas emocionais e de necessidades classificados como não urgentes (ZIMMERMANN LP et al., 2011, OLIVEIRA GN et al., 2011). Nesse contexto, este estudo teve por objetivo descrever o perfil da demanda em Unidades de Emergência Hospitalar, no ano de 2000 a 2019, segundo a literatura. 


\section{MÉTODOS}

Trata-se de um estudo de revisão de literatura, que consiste em ampla análise de publicações, com a finalidade de obter dados e conclusões sobre determinada temática. O Critério de inclusão artigos disponíveis na integra no período de 2000 até 2019. O critério de exclusão foi artigos duplicados.

Para elaboração dessa revisão foram seguidos quatro passos. Primeiramente, foram demarcadas as principais questões relacionadas ao perfil da demanda em Unidade de Emergência Hospitalar. No segundo passo, procedeu-se à busca nas bases de dados Biblioteca Virtual de Saúde (BVS), Scientific Electronic Library Online (SCIELO) e Literatura Latino-americana e do Caribe em Ciências da Saúde (LILACS).

Na primeira $1^{\text {a }}$ etapa utilizamos como pergunta norteadora:Qual o perfil epidemiológico da demanda em Unidades de Emergência Hospitalar ? na $2^{\underline{a}}$ etapa foi realizada uma busca ampla através de bases dados eletrônicas; na $3^{\text {a }}$ etapa houve a coleta de dados nas bases de dados ja citada anteriormente, buscando artigos publicados entre os anos de 2000 a 2019; na 4ª etapa ocorreu uma análise crítica dos estudos encontrados; na $5^{\text {a }}$ etapa, após a leitura e síntese dos resultados encontrados, foi realizada a análise a partir dos dados encontrado; e por último, a 6 ${ }^{\underline{a}}$ etapa foi feita a finalização da discursão dos resultados deste estudo.

\section{RESULTADOS}

A amostra desta revisão foi de 13 artigos expostos no Quadro 1. Os estudos foram publicados, em sua maioria, no ano 2009, e 2014 com duas publicações e nos anos de 2000, 2003, 2008, 2010, 2011, 2012, 2013, 2015 e 2019 com apenas uma publicação.

Quadro 1 - Quadro sinóptico dos artigos segundo títulos, autores, ano e revista.

\begin{tabular}{|c|c|c|c|}
\hline Artigo & Autores & Ano & Revista \\
\hline $\begin{array}{l}\text { Percepção das enfermeiras sobre a unidade de } \\
\text { emergência. }\end{array}$ & $\begin{array}{l}\text { ANDRADE } \\
\text { CAETANO } \\
\text { SOARES E }\end{array}$ & 2000 & Rev RENE \\
\hline $\begin{array}{l}\text { Os motivos de procura pelo Pronto Socorro Pediátrico de } \\
\text { um Hospital Universitário referidos pelos pais ou } \\
\text { responsáveis. }\end{array}$ & BATISTELA S et al & 2008 & $\begin{array}{l}\text { Ciências Biológicas e } \\
\text { da Saúde }\end{array}$ \\
\hline $\begin{array}{l}\text { Perfil epidemiológico e clínico de pacientes adultos jovens } \\
\text { admitidos na sala amarela do centro de trauma do hospital } \\
\text { de base do Distrito Federal }\end{array}$ & DE SOUZA et al. & 2019 & $\begin{array}{l}\text { Rev. Divulgação } \\
\text { Cientifica Sena Aires }\end{array}$ \\
\hline $\begin{array}{l}\text { Supervisão do Enfermeiro no Atendimento Pré-hospitalar } \\
\text { Móvel: visão dos auxiliares de enfermagem }\end{array}$ & BERNARDES A et al & 2009 & CiencCuid Saúde \\
\hline $\begin{array}{l}\text { Qualidade dos prontos-socorros e prontos-atendimentos: a } \\
\text { satisfação dos usuários }\end{array}$ & LIMA & 2015 & Rev. Einstein \\
\hline $\begin{array}{l}\text { Caracterização do perfil das emergências clínicas no } \\
\text { pronto-atendimento de um hospital de ensino }\end{array}$ & RIBEIRO RM et al & 2014 & Rev Min Enferm \\
\hline $\begin{array}{l}\text { Perfil dos pacientes atendidos na sala de emergência do } \\
\text { pronto socorro de um hospital universitário. }\end{array}$ & ROSA et al. & 2011 & Rev. Enferm. UFSM \\
\hline $\begin{array}{l}\text { Uma visão assistencial da urgência e emergência no } \\
\text { sistema de saúde }\end{array}$ & ROMANI HM et al & 2009 & Rev. Bioética, \\
\hline $\begin{array}{l}\text { Contributos psicológicos para a compreensão da utilização } \\
\text { inapropriada de um serviço e urgência pediátrica. }\end{array}$ & RODRIGUES SSOF & 2012 & $\begin{array}{l}\text { Rev. Enfermagem } \\
\text { Referencialll }\end{array}$ \\
\hline $\begin{array}{l}\text { O contexto de um serviço de emergência: com a palavra, o } \\
\text { usuário }\end{array}$ & $\begin{array}{l}\text { LUDWING MLM, } \\
\text { BONILHA ALL }\end{array}$ & 2003 & $\begin{array}{l}\text { Rev. Brasileira de } \\
\text { Enfermagem, }\end{array}$ \\
\hline $\begin{array}{l}\text { Perfil dos pacientes pediátricos atendidos na emergência de } \\
\text { um hospital universitário }\end{array}$ & $\begin{array}{l}\text { SALGADO RMP, } \\
\text { AGUERO FCM. }\end{array}$ & 2010 & Rev.Pediatria \\
\hline $\begin{array}{l}\text { As barreiras da universalidade do acesso vivenciado por } \\
\text { idosos nas unidades saúde da família }\end{array}$ & $\begin{array}{l}\text { SILVA } \\
\text { RABINOVICH EP }\end{array}$ & 2013 & $\begin{array}{l}\text { Rev.Eletrônica } \\
\text { Fainor }\end{array}$ \\
\hline $\begin{array}{l}\text { Caracterização do perfil assistencial dos pacientes adultos } \\
\text { de um pronto socorro }\end{array}$ & $\begin{array}{l}\text { OHARA R, MELO } \\
\text { MRAC, LAUS AM }\end{array}$ & 2010 & Rev.Bras Enferm \\
\hline $\begin{array}{l}\text { Perfil epidemiológico dos usuários do pronto atendimento } \\
\text { de um Hospital do Interior do Estado de Minas Gerais }\end{array}$ & $\begin{array}{l}\text { ALVES CFP, SILVA } \\
\text { ES, SILVA JV }\end{array}$ & 2014 & $\begin{array}{l}\text { Rev. Eletrônica Acervo } \\
\text { Saúde }\end{array}$ \\
\hline
\end{tabular}

Fonte: Pereira JA, Ruas JPP, Flausino VO, 2019. 
No que concerne ao local em que os artigos selecionados foram publicados, identificou-se na Revista RENE, Ciência Biológicas da Saúde, Revista Divulgação Cientifica, Sena Aires, Ciências, Cuidado em Saúde, Revista Einstein, Revista Mineira de Enfermagem, Revista Enfermagem UFSM, Revista Bioética, Revista Enfermagem Referencial, Revista Pediátrica, Revista Eletrônica Fainor, Revista Brasileira de Enfermagem e Revista Eletrônica Acervo Saúde cada uma com apenas uma publicação.

\section{DISCUSSÃO}

A partir das análises dos estudos encontrados, foi possível compreender e avaliar o perfil da demanda dos clientes que procuram atendimento no serviço de urgência e emergência. Compreende-se que o PS é um local destinado a um perfil de assistência a clientes com ou sem perigo de morte, cujos recursos à saúde inspiram a necessidade de atendimento rápido. Deve estar adequado para prestar assistência em situações de emergência - casos que precisam de atendimento imediato, porém, sem perigo de morte rápida e urgência - perigo iminente de morte (OHARA R, MELO MRAC e LAUS AM, 2010).

As características demográficas da maioria dos pacientes atendidos em emergências clínicas foram adultos jovens, gênero feminino, cor branca, casados e ativos no trabalho. As causas do atendimento mais prevalentes eram desconhecidas e as especialidades clínicas foram, respectivamente, neurologia, cardiologia e pneumologia. Os diagnósticos mais frequentes foram dor precordial, cefaléia e acidente vascular encefálico. $\mathrm{E}$ o destino final dos pacientes atendidos na emergência clínica foi em número maior a alta médica da unidade de pronto-atendimento da emergência, com pouca diferença entre os que internaram e posteriormente receberam alta hospitalar, sendo que $11,5 \%$ morreram. É importante caracterizar os pacientes, identificar as principais emergências clínicas, assim como o destino final (alta, óbito, internação), que envolvem o funcionamento de um serviço de pronto-atendimento da unidade de emergência a fim de auxiliar os profissionais de saúde no planejamento da assistência e, consequentemente, na melhoria do atendimento (RIBEIRO RM et al., 2014).

No estudo De Souza AMM et al (2019),foi encontro em seus resultados que a predominância do atendimento no pronto Socorro foi por traumas em clientes do gênero masculino, com $69,59 \%$, tendo com prevalência assistência medica por faixaetária de 20 a 24 anos, maiordemanda do serviço no period vespertino, com 35,33\%, com predominâncias de acidentes em via pública, com 74,50\% dos casos.

O pronto-socorro (PS) e as Unidades de Pronto Atendimento (UPA) são denominados como serviços de baixa resolutividade e qualidade, pois, são permeados por filas e tumulto. Soma-se a precariedade das condições materiais e tecnológicas; o número insuficiente de profissionais de saúde; a ausência de critérios de atendimento; a ausência de humanização e acolhimento dos trabalhadores de saúde com os usuários (BERNARDES A et al., 2009).

O descontentamento por parte dos pacientes pela assistência prestada foi devido alguns fatores, como por exemplo, assistência inapropriada, ausência de confiança com atendimento (LIMA CA et al.; 2015). O atendimento ocorrem em 24 horas no pronto socorro (SILVA GL e RABINOVICH EP, 2013).

A Política Nacional de atenção as urgência e emergência, mediante o quadro brasileiro de morbimortalidade relativo a relacionadas com trauma e violência estabelecem normas de organização do serviço privado e público de atenção terciária (BRASIL, 2002).

O AUE deverá estar adequadamente localizado com fácil acesso e terá de contar com equipe multiprofissional. O PS deve dispor de infraestrutura adequada com as demais salas: de recepção, de espera, de higienização, de serviço social, de gesso, de suturas, de curativos, de medicação, de observação, posto de enfermagem, sala procedimentos invasivos, sala para pequenas cirurgias e consultórios (ROMANI HM et al., 2009).

A atenção primaria deveriam ser a porta de entrada dos clientes ao sistema único de saúde disponibilizando uma assistência integral aos clientes, cabendo ao hospital ter recursos humanos e exames tecnológicos mais avançados e atender os casos de maior complicação. A inobservância desses critérios faz 
com que o serviço hospitalar tenha uma superlotação dos PS (RODRIGUES SSOF, 2012; BATISTELA S, GUERREIRO NP e ROSSETTO EG, 2008).

A busca pelo serviço de emergência deve acontecer a partir do referenciamento de cidadãos pelos profissionais de saúde, mas, na maioria dos casos, o paciente procura as unidades de urgência, sem antes ter buscado por atendimento na atenção básica. Todavia, as pessoas julgam seus problemas de saúde como dignos de atendimento de emergência, usando o serviço de forma incorreta (LUDWING MLM e BONILHA ALL, 2003).

A relação com o dia-a-dia do serviço nos leva a pensar na assistência em urgência e emergência e a sua necessidade de definição de novas práticas de saúde, elaborando elos de comunicação entre profissionais e clientes e consequentemente a todos os serviços ofertados. (BERNARDES A et al., 2009).

Segundo Rosa TP et al., (2011), no seu estudo que 74,5\% dos indivíduos atendidos em consultórios de Emergência de Pronto Socorro são do sexo masculino; de trinta e uma a sessenta anos (48,9\%); casados $(61,7 \%)$; com ensino fundamental incompleto $(70,2 \%)$, e procedentes de cidades próximas as unidades de PS, (53,4\%). O principal motivo do atendimento foi por trauma leve (31,9\%) e AVC (8,5\%); principalmente no turno da tarde (49\%) e $92 \%$ dos indivíduos atendidos continuaram internados por um tempo no pronto socorro.

Segundo o estudo de SALGADO RMP e AGUERO FCM (2010) afirma que a demanda por idade, duas faixas etárias, juntas predominam (De 20 á 39 anos e de 40 á 59 anos), concentram mais da metade dos atendimentos, à demanda por sexo, considerou-se o predomínio do sexo masculino, ocupando quase $60 \%$ dos atendimentos avaliados. Nos atendimentos por especialidades, em relação aos setores de Pequena Cirurgia, Clínica Médica e Traumatologia são as principais especialidades procuradas pelos usuários.

No que se refere ao motivo da procura por atendimento no pronto socorro de acordo com o estudo de Batistela S, Guerreiro NP e Rossetto EG (2008), foi identificado que: $41,3 \%$ devido à febre; $11,5 \%$ tosse; $7,9 \%$ vômito; $5,5 \%$ alergia; 5,3\% dor abdominal; 5,2\% tiveram algum tipo de queda; 4,9\% diarréia; 3,6\% tiveram algum tipo de trauma; $3,1 \%$ dor de ouvido, $2,2 \%$ cansaço; $1,9 \%$ cefaleia; $1,1 \%$ com coriza; $0,9 \%$ dor de garganta; $0,4 \%$ apresentando lesão com algum tipo de corpo estranho; $0,1 \%$ catapora e $0,1 \%$ efetuaram sutura.

Outros problemas de saúde mencionados foram relatados: constipação intestinal, choro. No Brasil, as patologias diarreicas foram à segunda causa de internação para menores de cinco anos, sucedida na rede de saúde pública e conveniada do SUS em 2001 (BRASIL, 2002).

Na pesquisa de Rodrigues SSOF (2012), há domínio de pedidos de exames radiográficos $(32,2 \%)$, aos laboratoriais (19,8\%), sendo que $80,2 \%$ das consultas não precisaram de exames laboratoriais para complementação diagnóstica e 67,8\% não precisara de radiografia para complementação diagnóstica.

No que se refere à atenção em urgência, o aumento do número de acidentes, a violência urbana e a estrutura precária da rede de atendimento de saúde são motivos que têm contribuído decisivamente para a sobrecarga dos serviços hospitalares. O conhecimento desta realidade em nosso meio é de relevância fundamental, no sentido de revelar a real situação do atendimento, para assim iniciar um processo de reestruturação do atual sistema de saúde, na perspectiva de consolidação dos princípios do SUS (ANDRADE LM, CAETANO JF e SOARES E, 2000).

Segundo o estudo de Rodrigues SSOF (2012), 60\% dos pacientes foram encaminhados para intervenção terapêutica na sua residência; $16,2 \%$ tiveram como designação a administração de hidratação via oral; $6,7 \%$ administração de medicação endovenosa; $2 \%$ colocação de gesso $15,1 \%$ não tiveram designação de qualquer tratamento.

A sociedade continua no sistema imediatista, desconhece as políticas de saúde e o fluxo do sistema de Urgência e Emergência, contribuindo com a modificação da assistência. Devido o modelo biomédico o pronto atendimentopasso a ser visto pela sociedade com porta de entrada consequentemente, tem o aumento dos gastos e sobrecarrega dos trabalhadores de saúde (ALVES CFP;SILVA ES e SILVA JV, 2014). 


\section{CONSIDERAÇÕES FINAIS}

Pode-se identificar com esta revisão de literatura que os motivos dos atendimentos nas unidades de emergência hospitalar, são constituídos de queixas de baixas complexidades, as quais, pela avaliação de Risco de Manchester, poderiam ser resolvidas na Unidade Básica de Saúde, uma vez que não condizem com o porte de uma unidade de urgência e emergência referenciada. Assim, a procura por esses serviços estão subestimadas pela população. Estudos futuros devem ser desenvolvidos, com abordagens metodológicas variadas, acerca dessa temática e que poderão subsidiar melhor planejamento e qualidade dos serviços de urgência e emergência.

\section{REFERÊNCIAS}

1. ANDRADE LM, CAETANO JF, SOARES E. Percepção das enfermeiras sobre a unidade de emergência. Rev RENE. V.1, n. 1, p. 91-7. 2000.

2. ALVES CFP, SILVA ES, SILVA JV. Perfil epidemiológico dos usuários do pronto atendimento de um Hospital do Interior do Estado de Minas Gerais. Rev. Eletrônica Acervo Saúde, 2014. V.5, n.2, p. 426-438, 2014.

3. BATISTELA S, GUERREIRO NP E ROSSETTO EG. Os motivos de procura pelo Pronto Socorro Pediátrico de um Hospital Universitário referidos pelos pais ou responsáveis. Ciências Biológicas e da Saúde, Londrina; 2008; 29(2): 121-130.

4. BERNARDES A et al. Supervisão do Enfermeiro no Atendimento Pré-hospitalar Móvel: visão dos auxiliares de enfermagem. CiencCuid Saúde, São Paulo, 2009 v 8,n.1, Jan/Mar. 2009.

5. BRASIL. Ministério da Saúde. Secretaria de Políticas de Saúde. Atenção integrada às doenças prevalentes na infância. Brasília; 2002.

6. DE SOUZA AMM et al. Perfil epidemiológico e clínico de pacientes adultos jovens admitidos na sala amarela do centro de trauma do hospital de base do Distrito Federal. Rev. Divulgação Cientifica Sena Aires. V.8, n.1, 2019.

7. GARLET ER et al. Finalidade do trabalho em urgências e emergências: concepções de profissionais. Rev. Latinoam Enfermagem, 2009.

8. GIL AC. Como elaborar projetos de pesquisa. 5.ed. São Paulo: Atlas, 2010. 175p.

9. LIMA CA et al. Qualidade dos prontos-socorros e prontos-atendimentos; a satisfação dos usuários. Rev. Einstein. V.13, n.4, p. 587-93. 2015.

10. RIBEIRO RM et al. Caracterização do perfil das emergênciasclínicas no pronto-atendimento de um hospital de ensino. Rev Min Enferm. V. 18, n. 3, p. 533-538. 2014.

11. ROSA TP et al. Perfil dos pacientes atendidos na sala de emergência do pronto socorro de um hospital universitário. Rev. Enferm. UFSM, V.1n.1, p. :51-60.2011.

12. ROMANI HM et al. Uma visão assistencial da urgência e emergência no sistema de saúde. Rev. Bioética,2009.

13. RODRIGUES SSOF. Contributos psicológicos para a compreensão da utilização inapropriada de um serviço e urgência pediátrica. Rev. Enfermagem Referenciallln.7 - julho 2012

14. LUDWING MLM, BONILHA ALL. O contexto de um serviço de emergência: com a palavra, o usuário. Rev. Brasileira de Enfermagem, Brasília, 2003; v. 56, n. 1, p. 12-17. 2003.

15. MALVESTIO MAA, SOUSA RMC. Suporte avançado a vida: atendimento a vitimas de acidentes de transito. Rev. Pública, São Paulo, v. 36, n. 5, p. 584-589, out, 2002.

16. PRAÇA W.R et al. Perfil epidemiológico e clínico de vítimas de trauma em um hospital do Distrito Federal, Brasil. Revista Pre Infec e Saúde. V.3, n.1, p. 1-7. 2017.

17. SALGADO RMP, AGUERO FCM. Perfil dos pacientes pediátricos atendidos na emergência de um hospital universitário. Rev.Pediatria, SÃO PAULO, 2010;32(2):90-7

18. SILVA GL, RABINOVICH EP. As barreiras da universalidade do acesso vivenciado por idosos nas unidades saúde da família. C\&D- Rev.Eletrônica da Fainor, Vitória da Conquista, v.6, n.1, p.3-24, jan./jun. 2013.

19. OHARA R, MELO MRAC, LAUS AM. Caracterização do perfil assistencial dos pacientes adultos de um pronto socorro. Rev.Bras Enferm, Brasília 2010 set-out; 63(5): 749-54.

20. OLIVATI FN et al. Perfil da demanda de um pronto-socorro em um município do interior do estado de São Paulo. Rev. RFO, Passo Fundo, v. 15, n. 3, p. 247-252, set./dez. 2010.

21. OLIVEIRA GN et al. Perfil da população atendida em uma unidade de emergência referenciada.Rev. Latino-Am. Enfermagem. Campinas,19(3):[09 telas] maio - jun2011.

22. ZIMMERMANN LP et al. Avaliação do grau de dependência de cuidados de enfermagem dos pacientes internados em pronto-socorro. Rev. Enferm. UFSM 2011 Mai/Ago;1(2):153-163. 